\title{
La instrucción basada en el procesamiento frente al enfoque ecléctico para el aprendizaje del subjuntivo en la enseñanza de E/LE
}

\author{
Irene Alonso Aparicio \\ Universidad de Granada \\ Recibido: 11-12-06 / Aceptado: 02-02-07 \\ ISSN: $1697-7467$
}

\begin{abstract}
RESUMEN: Este estudio plantea el doble objetivo de evaluar la eficacia de la instrucción formal en general y de dos propuestas de atención a la forma en particular: la instrucción basada en el procesamiento (IP) frente al enfoque ecléctico (EE). Para ello intervienen tres grupos de sujetos: uno que recibe clase bajo la IP, otro que recibe clase bajo el EE, y otro de control. La instrucción se centra en la distinción modal en construcciones temporales en español/lengua extranjera. Los resultados muestran rendimientos similares para ambas propuestas.
\end{abstract}

Palabras claves: instrucción formal, atención a la forma, instrucción basada en el procesamiento, enfoque ecléctico.

\begin{abstract}
The objective of the present research is twofold. First of all, we study the effects of classroom formal instruction; secondly, we evaluate the effects of a formfocused input-based approach, processing instruction (PI), versus the effects of a formfocused output-based approach, eclectic approach (EE). Participants included three sections: one section assigned to PI, one section assigned to EE, one section comprising a control group. Instruction lasted 5 days and targeted Spanish mood selection in temporal clauses. Results reveal similar gains for both approaches.

Key words: formal instruction, focus on form, processing instruction, eclectic approach.
\end{abstract}

\section{INTRODUCCIÓN}

No cabe duda de que a partir de cierta edad -primeros estadios de la adolescencia-, el proceso de adquisición de una lengua extranjera (LE) o segunda lengua (L2) requiere que se preste cierta atención a los aspectos formales del idioma meta desde la práctica docente. No obstante, no parece existir un consenso general acerca de las líneas didácticas que se deben adoptar. Las vertientes más radicales de los paradigmas principales de enseñanza de las últimas décadas -enseñanza de corte estructuralista o tradicional y enseñanza comunicativahan adoptado posturas opuestas, y sin embargo, ninguna se ha mostrado eficaz en el logro de una competencia de hablante nativo en el aprendiz. Por un lado, el proceder tradicional ha otorgado a la gramática una posición privilegiada -la corrección formal es crucial- y sin embargo, se ha mostrado ineficaz en el desarrollo de la competencia comunicativa. Por otro 
lado, aunque en el proceder comunicativo ha primado el desarrollo de la habilidad discursiva, se han advertido notables deficiencias formales en las producciones de los aprendices.

Práctica docente e investigación han puesto de manifiesto las limitaciones de ambas pedagogías pero han subrayado asimismo la necesidad de conjugar gramática y comunicación en el proceso de aprendizaje y enseñanza de una LE o L2. En este contexto, y con ánimo de conciliar las virtudes de ambas propuestas, Long (1991) propone la Atención a la Forma (Focus on Form $)^{1}$ como vía de tratamiento de la gramática:

La AF [Atención a la Forma] consiste, a pesar de sus diversas manifestaciones, fundamentalmente en que los aprendices conceden cierto tipo de atención a la forma lingüística para resolver una necesidad de contenido comunicativa; es decir, contando siempre con un contenido y uso evidentes o asequibles, dirigen su atención a cierto rasgo lingüístico necesario para lograr que el sentido avance o pueda establecerse. Ortega Olivares (2001:80).

A priori esta propuesta parece resolver el déficit de cualquier alternativa pedagógica previa, pero ¿en qué consiste realmente su puesta en escena?, ¿mediante qué estrategias de trabajo podemos armonizar gramática y comunicación? En esta línea de actuación situamos nuestra investigación. A continuación presentamos las bases teóricas de dos propuestas de trabajo de atención a la forma -o enfocadas en la forma-, y seguidamente las sometemos a evaluación. Concretamente evaluamos la eficacia de la instrucción basada en el procesamiento (VanPatten, 1996) -alternativa orientada al procesamiento del input o información lingüística a la que está expuesto el aprendiz- y para ello, la comparamos con el enfoque ecléctico -propuesta orientada al procesamiento del output o producción lingüística-. Con este propósito, elegimos el aprendizaje de la distinción modal en construcciones temporales por parte de estudiantes adultos de español como lengua extranjera (ELE).

Pretendemos con este trabajo extraer conclusiones que nos permitan contribuir al diseño de un modelo más eficaz y favorable en el tratamiento de la gramática en el aula de idiomas.

\section{Marco Teórico}

\subsection{La instrucción basada en el procesamiento}

Los antecedentes de la instrucción basada en el procesamiento ${ }^{2}$ (VanPatten, 1996) se sustentan en la creencia de que el desarrollo de la proficiencia en una LE o L2 es un proceso de adquisición que implica la creación de un sistema lingüístico implícito (inconsciente). No obstante, se reconoce que en dicho proceso la instrucción explícita puede contribuir de forma muy favorable.

${ }^{1}$ Surge el término Atención a la Forma frente a Atención a las Formas (tratamiento asociado con el proceder tradicional) y Atención al Significado (tratamiento asociado con el proceder comunicativo).

${ }^{2}$ La traducción es nuestra a partir del término Processing Instruction (VanPatten, 1996). 
Para el diseño de su propuesta pedagógica, VanPatten se apoya en su particular visión del proceso de adquisición de una segunda lengua (ASL), así como en la relevancia que en tal proceso tiene el input comprensible y significativo (información lingüística comprensible y significativa a la que está expuesto el aprendiz):

Figura 1: Modelo de ASL.

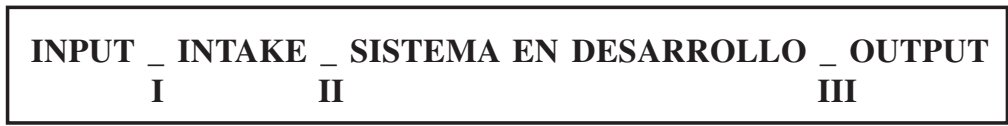

(Adaptado de VanPatten y Cadierno, 1993: 226)

De acuerdo con VanPatten, el proceso de ASL implica un conjunto de subprocesos que evolucionan en un único sentido (Figura 1). El primero de ellos, el procesamiento del input (I), se refiere a la percepción por parte del aprendiz de nuevo material lingüístico (input) y al procesamiento y retención en la memoria a corto plazo de parte de ese input (intake) durante la comprensión. El segundo subproceso, cambio del sistema (II), supone la acomodación del intake y la reestructuración del conocimiento implícito que tiene el aprendiz sobre la L2 (sistema lingüístico implícito en desarrollo). El tercer subproceso, procesamiento del output (III), se refiere al acceso al sistema en desarrollo y a la producción lingüística real (output).

Como se puede apreciar en la Figura 1, no cabe duda de que en este modelo de ASL "el desarrollo de un sistema interno depende del input"3 (Lee y VanPatten, 2003: 133), y es que, como mencionáramos anteriormente, el input y su procesamiento constituyen el grueso de la filosofía de la instrucción basada en el procesamiento (IP). Si tratamos de que el aprendiz llegue a producir output, será necesario alterar la naturaleza de su sistema en desarrollo, un cambio que podemos lograr trabajando la percepción y procesamiento del input. Luego, la IP surge como un modelo de instrucción destinado a operar sobre el primer subproceso del proceso de ASL -el procesamiento del input-pero, ¿en qué consiste ese procesamiento del input?, y ¿cómo podemos ayudar al aprendiz en esta labor para que obtenga gran cantidad de intake de calidad?

VanPatten describe el procesamiento del input como las estrategias psicolingüísticas en virtud de las cuales los aprendices extraen intake del input al que están expuestos, es decir, se trata de los mecanismos y estrategias mediante los que el aprendiz asigna significado a las formas lingüísticas del input cuando toda su atención se centra en la comprensión del mensaje que oye o lee ${ }^{4}$.

Sin embargo, cuando se afronta el aprendizaje de una LE o L2, no todas las estrategias de procesamiento resultan siempre necesariamente beneficiosas ${ }^{5}$. Pensemos por ejemplo en el

${ }^{3}$ La traducción es nuestra.

${ }^{4}$ Para una descripción detallada de los mecanismos y estrategias del procesamiento del imput, véase VanPatten (1996, 2002) y Lee y VanPatten (2003).

${ }^{5}$ Para un análisis de las consecuencias negativas que se derivan de las estrategias naturales de procesamiento del imput en el caso del ELE, véase Alonso Raya (2004). 
caso de un aprendiz anglófono de ELE o EL2 de nivel inicial, de acuerdo con la estrategia del primer nombre ${ }^{6}$, una instancia como (1) sería interpretada erróneamente como (2)

(1) La sigue el señor.

(2) * She follows the man.

* Ella sigue al señor.

Así, teniendo en cuenta las estrategias y mecanismos naturales de procesamiento del input, VanPatten propone la IP como un tipo de instrucción gramatical cuyo objetivo se convierte en "alejar a los aprendices de estrategias de procesamiento no óptimas [...] hacia tendencias más óptimas"8 (VanPatten, 2002: 764-765) para que extraigan con éxito la mayor cantidad posible de intake a partir del input al que están expuestos.

Pero, ¿cómo se extrapolan todas estas propuestas teóricas a la práctica del aula? En primer lugar, VanPatten propone llevar a cabo la instrucción en tres fases fundamentales:

1. Informar a los estudiantes de la forma gramatical meta.

2. Informar a los estudiantes de estrategias concretas de procesamiento del input que puedan afectar negativamente la asimilación de la forma meta durante la comprensión.

3. Fomentar que los estudiantes procesen la forma gramatical meta a través de actividades de input estructurado.

Observamos que para lograr un óptimo procesamiento del input se proponen como instrumento esencial las actividades de input estructurado. El objetivo de estas actividades se convierte en que el aprendiz realice conexiones entre forma y significado en el input que oye o lee. Luego, se trata de presentar a los aprendices un tipo de input manipulado en el que necesitan depender de la forma del ítem gramatical meta para obtener el significado de la instancia en la que se inserta.

Veamos un ejemplo para la concordancia de género en español':

${ }^{6}$ La estrategia del primer nombre establece que "los aprendices poseen una estrategia por defecto que asigna el papel de agente (o sujeto) al primer sintagma nominal que se encuentran en una frase" (VanPatten, 2002: 758).

${ }^{7}$ Ejemplo extraído de VanPatten y Cadierno (1993:228).

${ }^{8}$ La traducción es nuestra.

${ }^{9}$ Para una ejemplificación detallada de actividades de input estructurado en el caso de ELE, véase Alonso Raya (2003, 2004); Farley (2000); Lee \& VanPatten (2003); VanPatten, Lee, \& Ballman (2004). 


\section{Actividad: ¿Quién es?}

Tu profesor va a describir a una persona. Decide a quién describe y si estás o no de acuerdo:

1. a. __ David Letterman

b. ___ estoy de acuerdo

2. a. __ David Letterman

b. ___ estoy de acuerdo

3. a. __ David Letterman

b. ___ estoy de acuerdo
Madonna

no estoy de acuerdo

Madonna

no estoy de acuerdo

Madonna

no estoy de acuerdo

\section{(EL PROFESOR LEE)}

1. Es dinámica.

2. Es comprensivo.

3. Es reservada.

(Adaptado de Lee \& VanPatten, 2003)

En esta actividad, la atención del estudiante se dirige a la marca de género del adjetivo (ítem gramatical meta). Se trata de que el aprendiz dependa del rasgo gramatical meta para obtener el significado y poder completar con éxito la actividad.

Por último, y probablemente la característica más sobresaliente y polémica de esta alternativa pedagógica es la no producción de output durante la instrucción, lo que no implica su exclusión en la clase de gramática. De hecho, en numerosas ocasiones VanPatten y sus adeptos han manifestado que "una lección de gramática coherente es aquélla que lleva al estudiante desde procesar una característica gramatical en el input hasta acceder a ella en su sistema en desarrollo para crear output"10 (Lee \& VanPatten, 2003: 181) y que al igual que "el input es necesario para crear un sistema [...] el input no es suficiente para desarrollar la habilidad de usar el lenguaje en un contexto comunicativo" $" 1$ (Lee \& VanPatten, 2003: 168).

\subsection{El enfoque ecléctico}

Hemos convenido en seleccionar el enfoque ecléctico (EE) como alternativa para evaluar la eficacia de la IP por su concurrencia en la mayoría de los materiales curriculares consultados para la enseñanza actual de ELE.

A grandes rasgos, la metodología empleada actualmente en la enseñanza de ELE se caracteriza por concebir la lengua como "habilidad lingüística"; por no sustentarse en una teoría del aprendizaje concreta, sino en una serie de principios que describen qué fomenta el aprendizaje de una LE o L2; por, como su nombre indica, conciliar varias tendencias metodológicas, adoptando e integrando consecuentemente planteamientos que van desde el método estructural hasta el enfoque comunicativo; por presentar asimismo una gran flexibi-

${ }^{10}$ La traducción es nuestra.

${ }^{11}$ La traducción es nuestra. 
lidad en sus técnicas didácticas; por asignar al profesor el rol de guía y mediador del aprendizaje de su alumnado; por la organización y planificación de actividades acordes a las necesidades del alumnado; y por aspirar a que el estudiante aprenda a usar la lengua para comunicarse de forma comprensible y aceptable.

En lo que al tratamiento de la gramática se refiere, ésta se concibe como instrumento imprescindible para el desarrollo de la comunicación. La enseñanza de la gramática se lleva a cabo mediante la presentación explícita del ítem gramatical meta y la posterior ejercitación a través de actividades variadas. Para la presentación del ítem gramatical se suele emplear algún recurso visual o algún pequeño texto escrito con el fin de situar al aprendiz en el contexto de estudio. A continuación se presenta a los aprendices la información necesaria (siempre ajustada a su nivel y necesidades) sobre el ítem gramatical meta. Y por último, la práctica, que avanza en una secuencia de mayor a menor control, se lleva a cabo mediante actividades significativas y contextualizadas que persiguen la consolidación de estructuras y la fluidez.

\subsection{El modo en la interfase de la sintaxis y la semántica: las oraciones subordinadas temporales}

En una oración, los adjuntos temporales no oracionales y los oracionales expresan una circunstancia de tiempo del núcleo verbal:

\section{(3) Esta mañana mi primo ha subido las escaleras en tres minutos.}

En (3), la información temporal que expresa esta mañana hace referencia a cuándo ha subido mi primo las escaleras, mientras que en tres minutos informa del tiempo que ha tardado en hacerlo.

Siguiendo a García Fernández y Pérez Saldanya (en Bosque y Demonte, 1999) y teniendo en cuenta las aportaciones de Sánchez, Martín, y Matilla (2000), en el caso de los adjuntos temporales oracionales -fenómeno linguístico de nuestra investigación-, la oración subordinada adverbial temporal indica cuándo se realiza la acción expresada en la oración principal en relación con la que la misma oración subordinada expresa; es decir, se establece una relación temporal entre las acciones designadas por ambas oraciones. Esta relación depende del conector que introduce la oración subordinada y puede ser de simultaneidad (4), anterioridad (5), posterioridad (6) y para delimitar el momento en el que comienza o deja de ser efectiva la acción expresada en la oración principal (7):

(4) Juan llegó cuando yo estaba en la cocina.

(5) Carlos se fue antes de que se supiera la noticia.

(6) Le mandamos la carta después de saber que estaba aquí.

(7) Desde que vive en Alburquerque, no padece de los bronquios.

Conectores como cuando, mientras, al (mismo) tiempo que, siempre que, cada vez que, etc. denotan simultaneidad mientras que antes de que se emplea para denotar anterioridad y después de que, luego que, una vez que, al poco tiempo que, etc. indican posterioridad. Por último, desde que, hasta que, tan pronto como, etc. indican el momento en el que comienza o termina la acción expresada en la oración principal. 
En cuanto al modo que pueden exhibir las oraciones subordinadas adverbiales temporales $^{12}$, tanto el indicativo como el subjuntivo son aceptables. Sin embargo, la construcción con uno u otro modo implica una diferencia de interpretación normalmente referida como real/ irreal o factual/no-factual y depende de factores tanto semánticos como sintácticos. El modo indicativo se emplea cuando la oración subordinada tiene un carácter factual y hace referencia a un hecho experimentado (pasado, presente o habitual) (8); por el contrario, se emplea el modo subjuntivo cuando la oración subordinada tiene un carácter no-factual, es decir se refiere a una situación no experimentada, una situación posterior al momento que se toma como referencia. Esta idea de posterioridad se puede expresar mediante formas diversas como el futuro, condicional, imperativo, presente, "ir a + infinitivo", etc.; y además, puede referirse al momento del acto de habla (9) o a un momento del pasado (10):

(8) Visitó muchos museos mientras estuvo en Madrid.

(9) Díselo en cuanto puedas.

(10) Quería que me quedara hasta que pasasen las fiestas.

\section{Metodología}

\subsection{Objetivos}

Esta investigación plantea como objetivo general estudiar el impacto de la instrucción formal en el aprendizaje de la distinción modal en español en construcciones temporales y en tareas de interpretación y producción en el nivel oracional. Asimismo y de forma más específica, pretendemos estudiar la eficacia de la IP frente al EE.

A partir de las conclusiones extraídas, la finalidad última de este trabajo se convierte en la descripción de unas líneas de actuación didáctica para abordar la instrucción formal del modo más eficaz y favorable posible.

Proponemos, a modo de preguntas, las siguientes hipótesis de investigación:

- ¿Es la instrucción formal eficaz en la promoción del desarrollo de habilidades en el aprendiz para:

- Interpretar distinciones modales en español en las oraciones temporales?

- Producir distinciones modales en español en las oraciones temporales?

- ¿Qué alternativa metodológica de las estudiadas resulta más eficaz para:

- Interpretar distinciones modales en español en las oraciones temporales?

- Producir distinciones modales en español en las oraciones temporales?

Considerando las conclusiones obtenidas en trabajos previos que han centrado su atención en la eficacia de la IP frente a otras alternativas pedagógicas, predecimos que:

${ }^{12}$ Según las aportaciones de García Fernández y Pérez Saldanya (en Bosque y Demonte, 1999) y de Sánchez, Martín, y Matilla (2000). No hemos encontrado referencia alguna a la distinción modal en las construcciones temporales en la obra de Alcina Franch y Blecua (1980) ni en la de Gómez Borrego (1997). 
- La instrucción formal tiene un impacto positivo en el aprendizaje de la distinción modal en español en oraciones temporales tanto en tareas de interpretación como de producción.

- La eficacia de la IP y del EE en el aprendizaje de la distinción modal en español en las oraciones temporales será similar para las tareas de interpretación.

- La eficacia del EE en el aprendizaje de la distinción modal en español en las oraciones temporales será superior para las tareas de producción que la eficacia de la IP.

\subsection{Sujetos}

Un total de 30 estudiantes adultos de ELE tomaron parte en nuestra investigación. Los participantes se agruparon en tres grupos: grupo de control (no recibió instrucción formal sobre nuestro ítem gramatical), Grupo experimental 1 (recibió instrucción bajo la IP), y Grupo experimental 2 (recibió instrucción bajo el EE).

Los participantes tenían un nivel de lengua española intermedio ${ }^{13}$, habiendo estudiado nuestra lengua desde dos años el que menos hasta cuatro años el que más.

\subsection{Procedimientos de instrucción}

El periodo de instrucción para los dos grupos experimentales se extendió a lo largo de cinco sesiones lectivas de 50 minutos de duración cada una, repartidas en cinco días de clase.

El primer día de clase se dedicó a la realización del test inicial por parte de los tres grupos de trabajo (véase Apéndice A). Asimismo, se le hizo entrega a los grupos experimentales de un pequeño documento informativo con la explicación formal del ítem gramatical meta y una pequeña actividad a modo de deberes.

Durante el segundo y tercer día se impartieron clases a los grupos que recibían instrucción siguiendo las líneas metodológicas propuestas por la IP para el Grupo experimental 1 (véase Apéndice B) y siguiendo las pautas propuestas por el manual Avance (2001) para el Grupo experimental 2 (véase Apéndice C). El cuarto día se dedicó íntegramente a repaso y consulta de dudas. El grupo de control no recibió instrucción formal sobre el ítem gramatical meta a lo largo de estas sesiones.

Por último, el quinto día se les proporcionó a los tres grupos la misma versión del test inicial a modo de test final y se les pidió que lo volvieran a realizar.

\subsection{Instrumentos de evaluación}

El corpus de datos que ha servido de base para nuestro estudio consiste en un único test realizado a modo de pre-test y post-test (véase Apéndice A). Este test se compone de dos partes: una encuesta personal y una prueba experimental que incluye una tarea de interpretación y otra de producción de oraciones.

Mediante la encuesta personal se obtuvieron datos referentes al perfil de los informantes. En cuanto a la prueba experimental, el test incluyó una tarea de interpretación y otra de

${ }^{13}$ Equivalente al nivel B1 del Marco común europeo de referencia para las lenguas (2001). 
producción, ambas en el nivel oracional. La tarea de interpretación consistió en un juicio de gramaticalidad en el que se presentó a los aprendices un contexto y a continuación un conjunto de oraciones gramaticalmente correctas e incorrectas relacionadas con el contexto. Pedimos a nuestros estudiantes que decidieran si las oraciones eran gramaticalmente incorrectas y que detectaran, en el caso de serlo, dónde se encontraba la agramaticalidad. En cuanto a la tarea de producción, ofrecimos de nuevo un contexto a los estudiantes y seguidamente les presentamos un conjunto de oraciones incompletas para que las completaran rellenando los huecos con la forma correcta del verbo que aparecía entre paréntesis. Por último, se incluyeron en ambas tareas un total de 9 oraciones distractoras de las 25 que se presentaban para cada actividad.

\section{Resultados}

\subsection{Medias pre-test/post-test de los tres grupos en la tarea de interpretación}

A continuación presentamos los datos del comportamiento lingüístico de los tres grupos de trabajo en la tarea de interpretación antes (datos del pre-test) y después (datos del posttest) de la intervención docente.

Los datos de la tabla 1 y del gráfico 1 revelan una notable mejora en las habilidades de interpretación de los grupos que han recibido instrucción frente al grupo que no lo ha hecho. Concretamente, se ha obtenido un ascenso de la media para el Grupo Experimental 1 (GE1) desde 0,4 puntos en el pre-test hasta 7,2 puntos en el post-test, siendo el rango de valoración de 0 puntos para el no reconocimiento de frases agramaticales y 8 puntos para el completo reconocimiento de las mismas. De igual manera, la media para el Grupo experimental 2 (GE2) asciende de 0,2 puntos en el pre-test a 7,0 puntos en el post-test. Sin embargo, ninguna mejora se ha advertido en el Grupo de control (GC), que obtiene una media de 0,3 puntos en el pre-test y de 0,2 puntos en el post-test.

Tabla 1: Comparación de medias pre-test/pos-test en la tarea de interpretación.

\begin{tabular}{|c|c|c|}
\hline & Pre-test & Post-test \\
\hline GE1 & 0,4 & 7,2 \\
\hline GE2 & 0,2 & 7,0 \\
\hline GC & 0,3 & 0,2 \\
\hline
\end{tabular}


Gráfico 1: Comparación de medias pre-test/post-test en la tarea de interpretación.

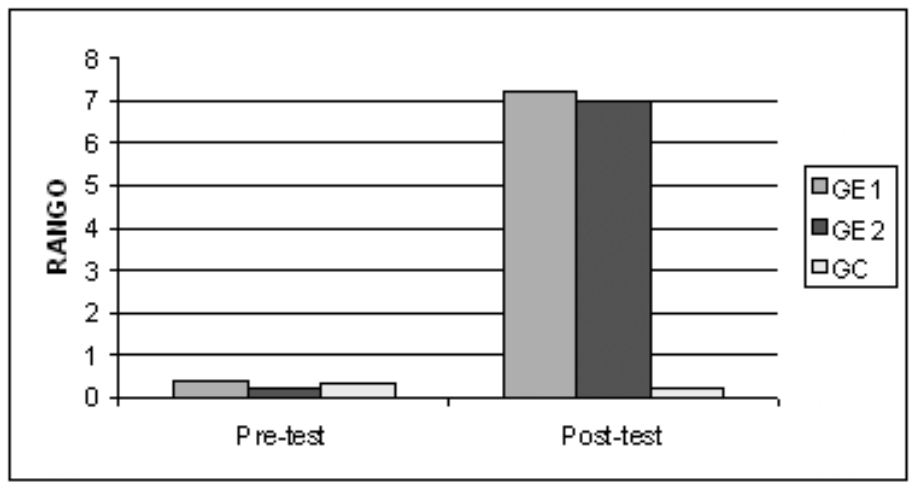

\subsection{Medias pre-test/post-test de los tres grupos en la tarea de producción}

Prestamos atención ahora a los datos obtenidos por los tres grupos de trabajo en la tarea de producción antes (datos del pre-test) y después (datos del post-test) de la intervención docente.

Al igual que ocurría en la tarea de interpretación, la tabla 2 y el gráfico 2 ponen de manifiesto una importante mejora en las habilidades de producción de los grupos que han recibido instrucción frente al grupo que no lo ha hecho. Concretamente, se ha obtenido un ascenso de la media para el Grupo Experimental 1 (GE1) desde 0,3 puntos en el pre-test hasta 11,9 puntos en el post-test, estando el rango de valoración establecido en 0 puntos para el $0 \%$ de respuestas correctas y 16 puntos para el $100 \%$ de respuestas correctas. De igual manera, la media para el Grupo experimental 2 (GE2) asciende de 0,2 puntos en el pre-test a 12,9 puntos en el post-test frente al estancamiento de las habilidades de aquellos sujetos que no han recibido instrucción formal sobre nuestro ítem gramatical (GC) con una media de 0,3 puntos en ambas pruebas.

Tabla 2: Comparación de medias pre-test/pos-test en la tarea de producción.

\begin{tabular}{|c|c|c|}
\hline & Pre-test & Post-test \\
\hline GE1 & 0,3 & 11,9 \\
\hline GE2 & 0,2 & 12,9 \\
\hline GC & 0,3 & 0,3 \\
\hline
\end{tabular}


Gráfico 2: Comparación de medias pre-test/post-test en la tarea de producción.

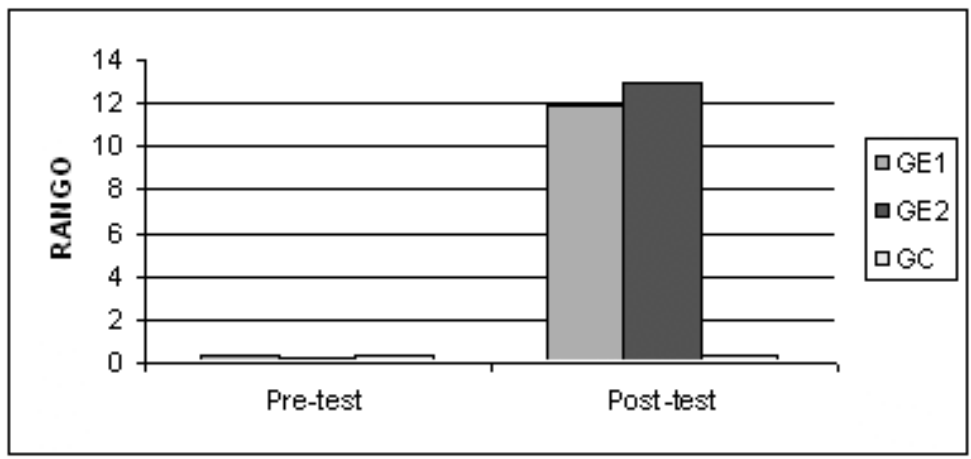

\subsection{Análisis estadístico}

Para comprobar la validez intrínseca de los métodos de instrucción utilizados -IP y EEse ha realizado una comparación entre los resultados obtenidos en el pre-test y post-test en cada una de las tareas propuestas (interpretación y producción). Presentamos a continuación los resultados.

\section{Instrucción vs. no instrucción en la tarea de interpretación}

Como se puede observar en la tabla 3, existen diferencias significativas entre los resultados sin instrucción y los resultados obtenidos con instrucción mediante el método de la IP en la tarea de interpretación (Sig.=0,000). De igual modo, en la tabla 4 se observan diferencias con significatividad estadística entre los resultados obtenidos con instrucción mediante el EE y los obtenidos por el grupo de control que no ha recibido instrucción expresa al respecto $(\mathrm{Sig} .=0,000)$. Es decir, la instrucción explícita sobre el ítem gramatical ofrece mejores resultados que la ausencia de instrucción para la tarea de interpretación.

Tabla 3: Prueba de muestras relacionadas en la tarea de interpretación entre la IP y la ausencia de instrucción.

\begin{tabular}{|c|c|c|c|c|c|c|}
\hline & \multicolumn{3}{|c|}{ Valor de prueba $=0$} \\
\hline & $\mathrm{T}$ & $\mathrm{gl}$ & Sig. (bilateral) & $\begin{array}{c}\text { Diferencia de } \\
\text { medias }\end{array}$ & \multicolumn{2}{|c|}{$\begin{array}{c}95 \% \text { Intervalo de } \\
\text { confianza para la } \\
\text { diferencia }\end{array}$} \\
\hline & & & & & Inferior & Superior \\
\hline diferencia & 21,000 & 9 &, 000 & 7,00000 & 6,2459 & 7,7541 \\
\hline
\end{tabular}


Tabla 4: Prueba de muestras relacionadas en la tarea de interpretación entre el EE y la ausencia de instrucción.

\begin{tabular}{|c|c|c|c|c|c|c|}
\hline & \multirow{2}{*}{} & & & \multicolumn{4}{|c|}{ Valor de prueba =0 } \\
& $\mathrm{T}$ & $\mathrm{gl}$ & Sig. (bilateral) & $\begin{array}{c}\text { Diferencia de } \\
\text { medias }\end{array}$ & \multicolumn{2}{c|}{$\begin{array}{c}95 \% \text { Intervalo de } \\
\text { confianza para la } \\
\text { diferencia }\end{array}$} \\
\hline & & & & & Inferior & Superior \\
\hline diferencia & 20,821 & 9 &, 000 & 6,80000 & 6,0612 & 7,5388 \\
\hline
\end{tabular}

Instrucción vs. no instrucción en la tarea de producción

En cuanto a la tarea de producción, nos encontramos con resultados similares. En la tabla 5 se aprecian diferencias significativas entre los resultados sin instrucción y los resultados obtenidos con la IP (Sig.=0,000). De forma análoga, en la tabla 6 se aprecian diferencias con significatividad estadística entre los resultados del EE y los obtenidos por el grupo de control (Sig.=0,000). Luego, la instrucción explícita sobre el ítem gramatical ofrece mejores resultados que la ausencia de instrucción para la tarea de producción.

Tabla 5: Prueba de muestras relacionadas en la tarea de producción entre la IP y la ausencia de instrucción.

\begin{tabular}{|c|c|c|c|c|c|c|}
\hline & \multicolumn{3}{|c|}{ Valor de prueba =0 } \\
\hline & $\mathrm{T}$ & $\mathrm{gl}$ & Sig. (bilateral) & $\begin{array}{c}\text { Diferencia de } \\
\text { medias }\end{array}$ & \multicolumn{2}{|c|}{$\begin{array}{c}\text { 95\% Intervalo de } \\
\text { confianza para la } \\
\text { diferencia } \\
\text { Inferior }\end{array}$} \\
\hline & Superior \\
\hline diferencia & 18,763 & 9 &, 000 & 11,60000 & 10,2014 & 12,9986 \\
\hline
\end{tabular}

Tabla 6: Prueba de muestras relacionadas en la tarea de producción entre el EE y la ausencia de instrucción.

\begin{tabular}{|c|c|c|c|c|c|c|}
\hline & \multicolumn{5}{|c|}{ Valor de prueba =0 } \\
\hline & $\mathrm{T}$ & $\mathrm{gl}$ & Sig. (bilateral) & $\begin{array}{c}\text { Diferencia de } \\
\text { medias }\end{array}$ & \multicolumn{2}{|c|}{$\begin{array}{c}\text { 95\% Intervalo de } \\
\text { confianza para la } \\
\text { diferencia }\end{array}$} \\
\hline & & & & & Inferior & Superior \\
\hline diferencia & 21,385 & 9 &, 000 & 13,60000 & 12,1614 & 15,0386 \\
\hline
\end{tabular}


La IP vs. el EE

Para evaluar qué método es mejor -IP o EE- en las tareas de interpretación y producción, se ha realizado un contraste de medias; para ello se ha creado una variable diferencia EE-IP, contrastando de este modo:

$$
\begin{gathered}
\mathrm{H}_{0}: \mathrm{EE}-\mathrm{IP}=0 \\
\mathrm{H}_{1}: \mathrm{EE}-\mathrm{IP} \neq 0
\end{gathered}
$$

No se puede afirmar la superioridad de ninguno de los dos métodos en ninguna de las dos tareas, pues la significatividad estadística es superior a 0,05 en ambos casos, concretamente, Sig. $=0,705$ en la tarea de interpretación (tabla 7), y Sig. $=0,066$ en la tarea de producción (tabla 8 ).

Tabla 7: Prueba de muestras relacionadas en la tarea de interpretación entre la IP y el EE.

\begin{tabular}{|c|c|c|c|c|c|c|}
\hline & \multicolumn{5}{|c|}{ Valor de prueba =0 } \\
\hline & $\mathrm{T}$ & $\mathrm{gl}$ & Sig. (bilateral) & $\begin{array}{c}\text { Diferencia de } \\
\text { medias }\end{array}$ & \multicolumn{2}{|c|}{$\begin{array}{c}\text { 95\% Intervalo de } \\
\text { confianza para la } \\
\text { diferencia }\end{array}$} \\
\hline & & & & & Inferior & Superior \\
\hline diferencia &,- 391 & 9 &, 705 &,- 20000 & $-1,3584$ &, 9584 \\
\hline
\end{tabular}

Tabla 8: Prueba de muestras relacionadas en la tarea de producción entre la IP y el EE.

\begin{tabular}{|c|c|c|c|c|c|c|}
\hline & \multicolumn{5}{|c|}{ Valor de prueba =0 } \\
\hline & \multirow{2}{*}{$\mathrm{T}$} & $\mathrm{Gl}$ & Sig. (bilateral) & $\begin{array}{c}\text { Diferencia de } \\
\text { medias }\end{array}$ & \multicolumn{2}{|c|}{$\begin{array}{c}95 \% \text { Intervalo de } \\
\text { confianza para la } \\
\text { diferencia } \\
\text { Inferior }\end{array}$} \\
\hline & Superior \\
\hline diferencia & 2,095 & 9 &, 066 & 2,00000 &,- 1593 & 4,1593 \\
\hline
\end{tabular}

\section{CONCLUSIONES E IMPLICACIONES DIDÁCTICAS}

Teniendo en cuenta los resultados que acabamos de presentar, son diversas las reflexiones que nos planteamos.

En lo que a la relevancia de la instrucción formal se refiere, el análisis del corpus de datos nos permite declarar su beneficio frente a la ausencia de instrucción en el aprendizaje a corto plazo de la distinción modal en oraciones temporales en ELE. 
En segundo lugar, los resultados nos permiten asimismo reflexionar sobre la validez de la IP como modelo de instrucción enfocada en la forma para concluir que dicha propuesta es eficaz, si bien, el similar comportamiento entre las alternativas pedagógicas propuestas no nos permite declarar su superioridad frente al EE.

Esta segunda conclusión contrasta con la alcanzada por el primer estudio que evaluó la eficacia de la IP (VanPatten \& Cadierno, 1993). En este estudio pionero, que midió el rendimiento de la IP frente a la instrucción formal tradicional ${ }^{14}$, se constató la superioridad de la propuesta de VanPatten ${ }^{15}$, unas conclusiones que se han visto repetidas en posteriores investigaciones ${ }^{16}$ que también han comparado ambas propuestas pedagógicas. Sin embargo, nuestros resultados se encuentran en consonancia con los hallazgos de diversos estudios que han evaluado la eficacia de la IP frente a pedagogías basadas en la producción significativa ${ }^{17}$ (donde se enmarcaría nuestra propuesta de contraste -el EE-).

Esta disparidad de resultados ha provocado que el debate en torno a la IP se centre en la definición y justificación de los elementos que intervienen y cómo lo hacen en el proceso de ASL, pues el rendimiento similar y en ocasiones superior de la instrucción basada en la producción significativa frente a la IP, ha sido interpretado por algunos estudiosos como signo inequívoco de que el output es asimismo responsable de la alteración del sistema en desarrollo del aprendiz.

No quisiéramos aquí entrar a discutir los componentes que alteran el sistema en desarrollo del aprendiz ni cómo lo hacen, pues quizás compete más a la psicolingüística que a nuestro objeto de estudio - una propuesta eficaz para el tratamiento de la gramática en el aula. No obstante, no podemos negar que la investigación ha cuestionado la teoría de ASL propuesta por VanPatten ${ }^{18}$.

De cualquier forma, retomando nuestro objeto de estudio, tampoco cabe duda de que "si los aprendientes no son expuestos a la lengua meta no podrán adquirirla" (Ellis, 2005: 42). Por consiguiente, se hace imprescindible un enfoque de actuación pedagógica que trabaje con esa lengua meta a la que están expuestos los aprendices. A este respecto, podemos afirmar que tanto nuestro estudio como estudios previos sobre la IP han puesto de manifiesto que las actividades de input estructurado contribuyen a la alteración del sistema en desarrollo del aprendiz y, por ende al desarrollo de su interlengua.

${ }^{14}$ Instrucción tradicional se refiere aquí a la presentación del ítem gramatical y su posterior práctica mediante ejercicios de producción oral y escrita que avanzan en una secuencia de carácter mecánica-significativa-comunicativa (VanPatten \& Cadierno, 1993).

${ }^{15}$ Los resultados mostraron que el grupo que recibió instrucción bajo la IP mejoró notablemente en sus habilidades de interpretación y producción mientras que el grupo que recibió instrucción tradicional sólo lo hizo en sus habilidades de producción. Estos resultados llevaron a VanPatten \& Cadierno (1993) a proclamar la superioridad de la IP frente a la instrucción tradicional; mientras la IP originaba un cambio en el sistema en desarrollo del aprendiz, mediante la instrucción tradicional el estudiante aprendía a realizar una actividad.

${ }^{16}$ Véase Cadierno (1995) y Benati (2001).

${ }^{17}$ Se trata de propuestas de instrucción orientadas a la producción oral y escrita que han eliminado el componente mecánico de la instrucción tradicional. Algunos de estos estudios incluyen: Salaberry (1997), Collentine (1998), Farley (2000), Toth (2006), Morgan-Short \& Wood Bowden (2006).

${ }_{18}$ Un asunto a su vez sobre el que aún no se ha alcanzado un acuerdo general en el campo de la Adquisición de Segundas Lenguas. 
Al margen de si la enseñanza basada en el procesamiento del input es o no superior [a la práctica de la producción], la investigación muestra, en efecto, que optar por un input estructurado resulta efectivo para promover el aprendizaje si éste es calibrado en términos de comprensión o de producción. Esta conclusión invita a considerar la necesidad de revisar los enfoques actuales de la enseñanza de la gramática para hacer un uso completo de esta posibilidad. Ellis (2005: 18).

Incluso aquéllos que con más crudeza han cuestionado la teoría de ASL de VanPatten, han tenido unas palabras de reconocimiento para su propuesta:

Bill VanPatten ha hecho una contribución muy importante a este campo de estudio [Adquisición de Segundas Lenguas] al llamar la atención sobre la importancia de dar a los estudiantes actividades que les lleven a procesar relaciones de forma y significado cruciales, en particular, actividades de comprensión. DeKeyser et al. (2002: 820$)^{19}$

Por consiguiente, a partir de este consenso general sobre la capacidad de la IP para contribuir favorablemente al desarrollo de la interlengua del aprendiz, animamos desde aquí y a partir de una perspectiva de enseñanza flexible, armoniosa y conciliadora, a que personal docente y creadores de material didáctico y currículos contemplen su inclusión en el aula con seguridad de un óptimo funcionamiento. Asimismo, invitamos a su consideración por las razones que a continuación se especifican.

En primer lugar, dado que la enseñanza está hoy más centrada que nunca en el aprendiz y sus necesidades, nos parece clave brindar al estudiante la oportunidad de asimilar el contenido formal meta antes de pedirle que lo produzca, pues desde la experiencia docente podemos corroborar el sentimiento de frustración que a menudo provoca una producción precipitada. En este sentido, no se nos ocurre mejor propuesta que las actividades de input estructurado.

Por otro lado, considerando la naturaleza de las actividades de input estructurado, no cabe duda de que éstas pueden fácilmente trabajarse fuera del aula. Luego, se presentan como una opción a considerar en aquellos cursos de lengua que cuentan con pocas horas lectivas de clase. Mientras las horas presenciales pueden dedicarse a la realización de actividades de producción, los contenidos gramaticales meta pueden asimilarse mediante las actividades de input estructurado a modo de deberes que además, pueden fácilmente editarse en soporte electrónico.

“¿Puede y debería la IP tener lugar fuera del aula, como deberes por ejemplo?, ¿necesita que se lleve al aula?, puesto que la IP se basa en el input, ¿se puede trabajar la IP de forma efectiva mediante el uso de ordenadores?, preguntas de este tipo ayudarán a profesores y creadores de proyectos curriculares a maximizar el uso de un lenguaje comunicativo durante las generalmente escasas sesiones de clase que los estudiantes de lengua pasan en el aula"20. VanPatten (1996: 158)

${ }^{19}$ La traducción es nuestra.

${ }^{20}$ La traducción es nuestra. 
Finalmente, no hemos de olvidar que "una lección de gramática coherente es aquélla que lleva al estudiante desde procesar una característica gramatical en el input hasta acceder a ella en su sistema en desarrollo para crear output" ${ }^{21}$ (Lee \& VanPatten, 2003: 181). Luego, si consideramos el similar rendimiento de nuestros dos grupos experimentales aun cuando el grupo de la IP no ha experimentado la fase de producción, de esa "lección de gramática coherente", ¿qué resultados se pueden llegar a obtener cuando ambas fases de la "lección de gramática coherente" se lleven a cabo? A priori, la respuesta resulta muy esperanzadora.

Partiendo de que "dadas las oportunidades adecuadas, niños mayores, adolescentes y adultos pueden y aprenden mucho de la gramática de una L2 mientras, dicho sea de paso, se centran en el significado, o la comunicación"22 (Long, 1997:1), invitamos a la consideración de la instrucción basada en el procesamiento como un valioso complemento al modus operandi convencional de la instrucción formal.

\section{Bibliografía}

Alcina Franch, J., Blecua J. M. (1980). Gramática Española. Barcelona: Ariel.

Alonso Raya, R. (2003). "Cómo cambiar tu vida con la gramática. Algunos consejos para tener éxito con los ejercicios gramaticales". XII Encuentro Práctico de Profesores de ELE. Barcelona: International House Barcelona y Difusión.

Alonso Raya, R. (2004). "Procesamiento del input y actividades gramaticales". Revista Electrónica de Didáctica/Español Lengua Extranjera, 0.

Benati, A. (2001). "A comparative study of the effects of processing instruction and output-based instruction on the acquisition of the Italian future tense". Language Teaching Research, 5, 2: $95-127$

Bosque, I., Demonte, V. (1999). Gramática descriptiva de la lengua española. Real Academia Española. Colección Nebrija y Bello. Madrid: Espasa-Calpe.

Cadierno, T. (1995). "Formal instruction from a processing perspective: an investigation into the Spanish past tense". The modern Language Journal, 79, 2: 179- 193.

Cerrolaza, O., Llovet, B., Cerrolaza, M. (1998). Planet@ ELE. Madrid: Edelsa.

Collentine, J. (1998). "Processing Instruction and the subjunctive". Hispania, 81, 3: 576-587.

DeKeyser, R. M., Salaberry, M. R., Robinson, P., \& Harrington, M. (2002). "What gets processed in processing instruction? A commentary on Bill VanPatten's "Processing instruction: An update". Language Learning, 52, 4: 805- 823.

Ellis, R. (2005). "La adquisición de segundas lenguas en un contexto de enseñanza. Análisis de las investigaciones existentes". Informe al Ministerio de Educación. Auckland Uniservices Limited.

Farley, A. P. (2000). The relative effects of processing instruction and meaning-based output instruction on L2 acquisition of the Spanish subjunctive. University of Illinois at UrbanaChampaign (tesis doctoral inédita).

Gómez Borrego, L. (1997). Gramática didáctica del español. Madrid: Ediciones SM.

Lee, J. F., VanPatten, B. (2003). Making communicative language teaching happen. New York: McGraw-Hill.

Long, M. (1991). "Focus on form: A design feature in language teaching methodology", en K. de Bot, R. Ginsberg \& C. Kramsch (eds.), Foreign language research in cross-cultural perspective. Amsterdam: John Benjamin, 39-52. 
Long, M. (1997). "Focus on form in Task-Based Language Teaching", Fourth Annual McGrawHill Satellite teleconference: Grammar in the Communicative Language Classroom.

Moreno García, C., Moreno Rico, V., Zurita P. (2001). Avance Intermedio. Madrid: SGEL.

Morgan-Short, K., Wood Bowden, H. (2006). "Processing instruction and meaningful output-based instruction: Effects on Second Language Development". Studies in Second Language Acquisition, 28, 1: 31-65.

Ortega Olivares, J. (2001). "Gramática y Atención a la Forma en el aula de E/LE". Revista del Instituto Cervantes de Nápoles, 1: 73-87.

Richards, J. C., Rodgers, T. S. (2001). Approaches and Methods in Language Teaching. Cambridge: Cambridge University Press.

Salaberry, M. R. (1997). "The role of input and output practice in second language". Canadian Modern Language Review,53,1: 422- 451.

Sánchez, A., Martín E., Matilla, J.A. (2000). Gramática práctica de español para extranjeros. Madrid: Sociedad General Española de Librería, S.A.

Trim, J. L. M., Coste, D., North, B., Sheila, J. (2001). Marco común europeo de referencia para el aprendizaje, la enseñanza y la evaluación de lenguas. Consejo de Europa.

Toth, P. D. (2006). "Processing Instruction and a Role for Output in Second Language Acquisition". Language Learning, 56, 2: 319- 385.

VanPatten, B. (1996). Input processing and grammar instruction: Theory and research. Westport, CT: Ablex.

VanPatten, B. (2002). "Processing Instruction: An update". Language Learning, 52, 4: 755-803.

VanPatten, B., \& Cadierno T. (1993). "Explicit instruction and input processing". Studies in Second Language Acquisition, 15, 2: 225-243.

VanPatten, B., Lee, J. \& Ballman, T. (2004). ¿Sabias que...? Beginning Spanish (4th ed.). Boston: McGraw-Hill. 


\section{APÉNDICE A}

Muestra adaptada del test

Nombre:

Edad:

Nacionalidad:

Sexo:

\section{TAREA DE INTERPRETACIÓN}

Presta atención a la siguiente viñeta e imagina un posible diálogo entre la pitonisa y Pedro:

¿Qué le deparará el futuro a Pedro?

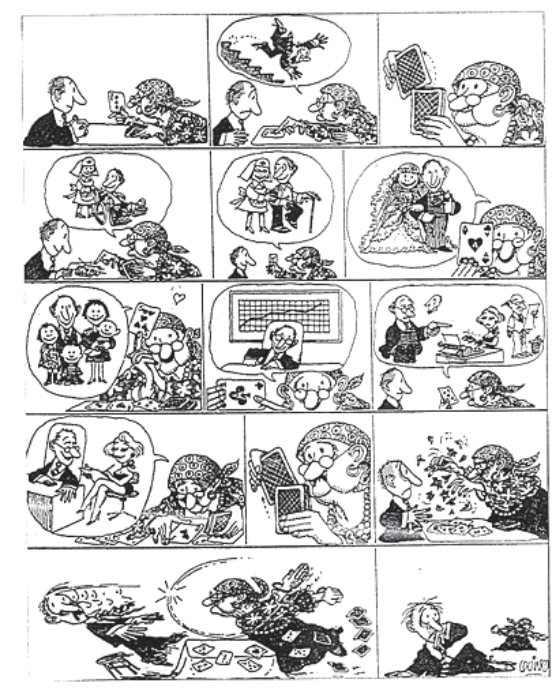

Adaptado de Planet@ELE (1998)

La pitonisa y Pedro han estado hablando sobre el posible futuro de Pedro. Aquí presentamos algunas de los comentarios que han hecho. Decide si las siguientes oraciones son o no gramaticalmente correctas y subraya la parte de la oración que no es gramatical:

1. Pedro: “¿Cómo será mi futuro mientras viva en los próximos años?"

2. Pitonisa: "Vas a tener una vida llena de aventuras".

3. Pitonisa: "Para empezar, un buen día, mientras bajas las escaleras de tu casa, te tropezarás y te caerás."

4. Pedro: “¿Me pasará algo grave cuando me caigo?” 
5. Pitonisa: "No, no será una caída grave pero tendrás que ir al hospital".

6. Pitonisa: "Cuando entras en el hospital, te atenderá una enfermera muy amable y guapa".

7. Pedro: “¿Me atenderá esa enfermera mientras me recupere?”

\section{TAREA DE PRODUCCIÓN}

Lee el siguiente texto sobre los planes de fin de semana que Susana ha preparado para Lucía.

\section{El fin de semana de Susana y Lucía}

Susana y Lucía son compañeras de piso. Hoy es viernes y acaban de terminar su primer trimestre en la universidad. Susana está triste porque no va a ver a su amiga durante tres semanas, así que ha preparado un plan divertido para pasar el último fin de semana del año juntas. Susana ha pensado que como hoy llueve mucho, sería una buena idea ir al cine a la sesión nocturna para ver una película cómica. Después del cine hay una fiesta de navidad en casa de unos amigos, así que si no llueve mucho, irán a despedirse de ellos. Para el sábado, Susana ha pensado que puede ser divertido ir las dos juntas a comprar los últimos regalos de navidad. Pasarán todo el día de compras y almorzarán en su restaurante italiano favorito, Giovani's. Por la noche, las dos irán a la fiesta de otros amigos con Manuel, un buen amigo de Susana que Lucía no conoce. El domingo prepararán sus maletas por la mañana y a las 2:00 de la tarde tomarán el autobús para sus casas.

Susana y Lucía han estado hablando durante horas esta tarde. Aquí presentamos parte de la conversación que han tenido. Completa las oraciones con la forma correcta del verbo:

Susana: Oye Lucía, ¿qué planes tienes para el fin de semana?

Lucía: No ___ (saber/yo). No he pensado nada.

Susana: ¡Perfecto! Yo lo he pensado todo.

Lucía: ¡Ah!, ¿sí?, y ¿qué haremos antes de que nos (ir/nosotros) el domingo?

Susana: Después de que (terminar/ tú) con tus clases esta noche, iremos al cine para ver una película cómica.

Lucía: ¡Vale! Pero, ¿vamos a ir a despedirnos de Juan y el resto antes de que (ir/nosotros) al cine?

Susana: Pues no sé, quizás no hay mucho tiempo. Si (tener/nosotros) tiempo podemos ir, y si no, esta noche organizan una fiesta, así que podemos ir a verles después de que (salir/nosotros) del cine. 


\section{APÉNDICE B}

\section{Muestra adaptada de las actividades del grupo IP}

\section{ACTIVIDAD A: LA VIDA DE ANTONIO GARCÍA MORAL}

A continuación presentamos algunas actividades sobre el pasado, presente y futuro de la vida de Antonio García Moral. Decide y marca con una X la opción correcta para comenzar cada frase:

1. ... recojo a mi hijo del colegio.

Cuando termino de trabajar,

___ Cuando terminé de trabajar,

2. ... iremos de vacaciones mi familia y yo a la costa.

En cuanto llega el verano,

___ En cuanto llegue el verano,

3. ... fui a vivir a Madrid.

Cuando acabe mi carrera en la universidad,

___ Cuando acabé mi carrera en la universidad,

4. ... mi mujer y yo compraremos una casa a las afueras de la ciudad.

Después de que vendamos nuestra casa actual,

Después de que vendemos nuestra casa actual,

5. ... hacía mucho deporte, especialmente natación.

Cuando era joven,

Cuando sea joven,

\section{ACTIVIDAD B: TU PROFESOR...}

Ahora te presentamos algunos detalles sobre la vida de tu profesor. Escucha atentamente a tu profesor y marca con una X la opción más apropiada:

1. ... lo llevaré al taller porque no sé nada de mecánica.

... lo llevo al taller porque no sé nada de mecánica.

2. ... me iré de vacaciones con mis amigas.

... me iba de vacaciones con mis amigas.

3. ... me acordé de Manuela.

... me acuerdo de Manuela.

4. ... paso el verano en la playa porque tengo mucho tiempo.

... pasaba el verano en la playa porque tenía mucho tiempo. 
5. ... plantaré rosas en el jardín.

... planto rosas en el jardín.

(EL PROFESOR LEE)

1. Cuando tenga algún problema con el coche,

2. Después de que termine este trabajo,

3. Siempre que paso por Bilbao,

4. Cuando era joven,

5. Tan pronto como llegue el buen tiempo, 


\section{APÉNDICE C}

\section{Muestra adaptada de las actividades del grupo EE}

[Material adaptado de Avance Intermedio (2001)]

\section{TERMINA LAS FRASES}

1. En cuanto me iré a dar una vuelta.

2. Cuando. pienso pasarme todo el mes sin hacer nada.

3. Oye, antes de que (tú). , tenemos que hablar seriamente.

4. Cuando. me compré unas botas de cuero baratísimas.

5. Te esperaré aquí hasta que.

6. Después de que (vosotros). las cosas se aclararon.

7. No pienso salir hasta que

\section{REACCIONA}

1. Mamá, ¿cuándo vas a comprarme los pantalones vaqueros?

Cuando

2. Yo nunca tomo aspirinas.

Pues yo las tomo cuando

3. ¿Cuándo sueles estudiar?

Cuando

4. ¿Cuándo vais a iros de vacaciones?

No sé, nos iremos cuando

5. ¿Vais a venir a comer a casa?

Sí, en cuanto

6. ¿Hasta cuándo estarás aquí?

Hasta que

7. ¿Puedo pasarme por tu casa?

Claro que sí, cuando

PARA ACLARAR LAS COSAS:

* SOLER = sólo se usa en presente e imperfecto

* PASARME POR TU CASA = ir a tu casa 